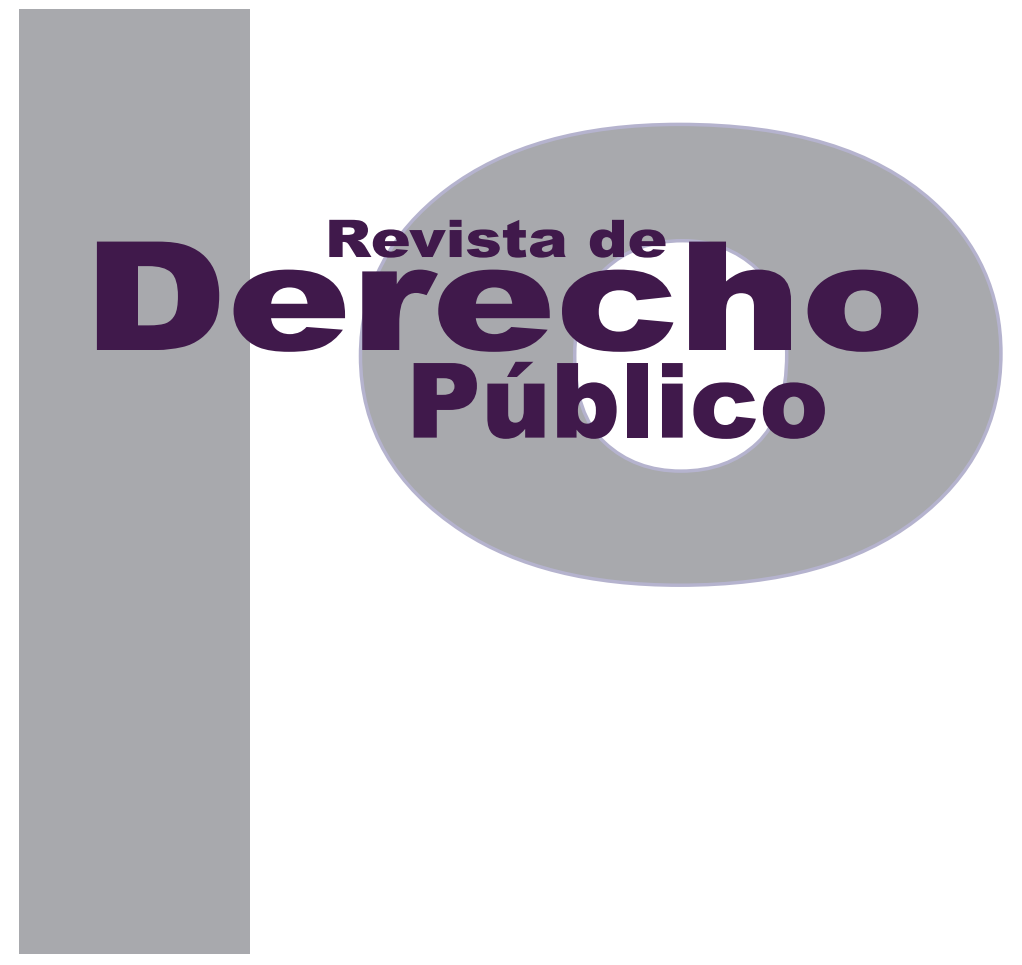

\title{
ESTUDIOS SOBRE LA VIOLENCIA INTRAFAMILIAR EN COLOMBIA: EL TRATAMIENTO DESDE LA PERSPECTIVA DE SALUD FÍSICA Y MENTAL
}

\author{
Manuela Piza \\ Revisión de Tema \\ DOI: http://dx.doi.org/10.15425/redepub.33.2014.32 \\ Universidad de los Andes \\ Facultad de Derecho \\ Revista de Derecho Público N. ${ }^{\circ} 33$ \\ Julio - Diciembre de 2014. ISSN 1909-7778
}




\title{
Estudios sobre la violencia intrafamiliar en Colombia: el tratamiento desde la perspectiva de salud física y mental
}

\section{Resumen}

Una buena parte de los estudios realizados en Colombia sobre violencia interpersonal contra mujeres adultas se refiere al problema de la recuperación de la salud mental y física de las afectadas y de sus agresores. La literatura existente sobre este tema, realizada en Colombia y con casos colombianos, se refiere a dos subtemas principales: la prevención de la violencia intrafamiliar (VIF) y el tratamiento posterior a los actos violentos. En cuanto a su enfoque, las fuentes se distinguen por su aproximación al tema: un primer grupo de fuentes asume una postura normativa/evaluativa, planteando las falencias del sistema de atención y las recomendaciones que se deben tener en cuenta para solucionarlas; el segundo grupo, por otra parte, asume una postura descriptiva/explicativa y se orienta a explicar los actores involucrados en el fenómeno, la prevención de la violencia interpersonal o tratamientos exitosos. Las tres conclusiones principales son: el precario desarrollo en la literatura sobre el tema, los problemas éticos a los que se enfrenta el personal de salud y los vacíos que impiden que haya una atención integral.

Palabras clave: violencia intrafamiliar, tratamiento, salud mental y física, agresores, agredidos, personal de salud.

\section{Studies about domestic violence in Colombia: the treatment from perspective of physical and mental health}

\begin{abstract}
A significant number of studies conducted in Colombia on interpersonal violence against adult women focus on the recovery of their mental and physical health and that of their partners. Existing literature on this topic, published in Colombia about research conducted in Colombia, clusters around two issues: prevention and treatment. It exhibits two different approaches as well: some papers and books adopt a normative/evaluative approach, proposing the failures in the system and recommendations as to how to fix it; other papers and books adopt a descriptive/explicative approach, explaining the constellation of actors involved in the phenomenon, the prevention of interpersonal violence and treatment of mental and physical disorders caused by it. The paper concludes that existing literature is scarce; that there is a need to attend the ethical problems confronted by health professionals in this area; and that many gaps in existing legislation prevent providing victims with a comprehensive health attention.
\end{abstract}

Keywords: domestic violence, treatment, mental and physical health, health staff, aggressors, assaulted.

\section{Estudos sobre a violência intrafamiliar na Colômbia: o tratamento desde a perspectiva de saúde física e mental}

\section{Resumo}

Uma boa parte dos estudos realizados na Colômbia em matéria de violência interpessoal contra mulheres adultas se refere ao problema da recuperação da saúde mental e física das afetadas e de seus agressores. A literatura existente sobre este tema, realizada na Colômbia e com casos colombianos, se refere a dois principais subtemas: a prevenção da violência intrafamiliar (VIF) e o tratamento posterior aos atos violentos. Sobre seu enfoque, as fontes se distinguem por sua aproximação ao tema: um primeiro grupo de fontes assume uma postura normativa/avaliativa, planteando as falências do sistema de atenção e as recomendações a ser levado em consideração para solucioná-las; o segundo grupo, de outra parte, assume uma postura descritiva/explicativa e se orienta a explicar os atores envolvidos no fenômeno, a prevenção da violência interpessoal ou tratamentos de sucesso. As três principais conclusões são: o precário desenvolvimento na literatura sobre o tema; os problemas éticos aos que se enfrenta o pessoal de saúde e os vazios que impedem que tenha uma atenção integral.

Palavras-chave: violência Intrafamiliar, tratamento, saúde mental e física, agressores, agredidos, pessoal de saúde. 


\title{
Estudios sobre la violencia intrafamiliar en Colombia: el tratamiento desde la perspectiva de salud física y mental ${ }^{* *}$
}

\author{
Manuela Piza**
}

\section{SUMARIO}

Introducción - I. ETAPAS - A. Primera etapa: La prevención - B. Segunda etapa: El tratamiento posterior al hecho violento - 1. Prediagnóstico e intervención inicial - 1.1. El personal de los centros de atención frente a los agresores - 1.2. El personal de los centros de atención frente a las víctimas 1.3. El personal de los centros de atención frente a los espectadores - 2. Nivel terapéutico - 3. Nivel especializado - 3.1. El caso particular de la ruta de atención en violencia sexual - II. CONCLUSIONES. Bibliografía.

* Cómo citar este artículo: Piza, M. (Diciembre, 2014). Estudios sobre la violencia intrafamiliar en Colombia: el tratamiento desde la perspectiva de salud física y mental. Revista de Derecho, Comunicaciones y Nuevas Tecnologías, 12. Universidad de los Andes (Colombia). ** Profesora asociada de la Facultad de Derecho de la Universidad de los Andes; directora del Observatorio del Proceso de Restitución de Tierras — territorio sur— de la Facultad de Derecho de la misma universidad. Correo: pmoncada@uniandes.edu.co

*** Abogada de la Universidad de los Andes y miembro del grupo de investigación de Derecho y Género. Correo electrónico: manuelapizac@gmail.com 
Introducción

Una de las áreas que se han investigado en relación con el tema de violencia intrafamiliar (VIF) en Colombia es la de la salud mental y física, tanto de los agresores como de los agredidos. Dentro de esta hay dos elementos importantes: por un lado, los trastornos asociados con este tipo de violencia y, por otro, el tratamiento que se le da a las dos partes involucradas en el conflicto. El presente trabajo pretende ocuparse de este último elemento.

Para analizar el estado del arte del tratamiento de VIF en Colombia es importante partir desde la determinación de los ejes sobre los que se ha desarrollado la investigación. Un primer eje divide la literatura existente en dos; la que se refiere a los tratamientos que buscan la prevención de la VIF y la que se refiere a los tratamientos posteriores a los actos violentos dentro de la familia. El segundo eje, que se ocupa de los actores (agresores, los agredidos, los espectadores y el personal de salud), ha sido desarrollado tanto en la etapa de prevención como en la etapa de tratamiento posterior.

El presente documento hará primero unas aclaraciones generales sobre el tratamiento de VIF en salud física y mental para luego tratar cada uno de los ejes mencionados de manera más detallada. El análisis ulterior se hará sobre la etapa previa y posterior al hecho violento $y$, en cada una de las etapas que lo componen, se analizarán los actores relevantes que participan en él. Por último, se presentarán unas conclusiones sobre el estado del arte de los tratamientos en salud física y mental de la VIF.
Antes de ahondar en cada uno de estos ejes es importante hacer unas aclaraciones generales. Para el desarrollo de este trabajo se hizo un análisis de 47 fuentes bibliográficas. La búsqueda se hizo principalmente a través de Google, Google Scholar y de los buscadores de las bibliotecas de la Universidad de los Andes, la Universidad de Antioquia, la Universidad Externado de Colombia y la Universidad Nacional, utilizando los términos "violencia intrafamiliar" y "tratamiento en salud física y mental". De esta búsqueda, el mayor número de fuentes útiles para el presente documento se obtuvieron de la base de datos de la Universidad de Antioquia.

De la primera selección que se hizo se descartaron 24 fuentes porque no contenían elementos relevantes sobre el tema. De las 23 restantes, 3 son tesis de grado, 6 artículos de revista, 7 documentos oficiales y 7 libros, las cuales se pueden dividir en dos grupos. Por un lado, aquellas que plantean las falencias de la situación a grandes rasgos, es decir, que se centran en la poca investigación e información que hay respecto al tema y hacen algunas recomendaciones sin mucho desarrollo, recomendaciones importantes pero que más allá de evidenciar el problema no dan ideas ni estructuras para el perfeccionamiento. Esto se debe a que la mayor parte de estas recomendaciones están en fuentes que se ocupan del problema de la VIF en general y de manera más cuantitativa. Por otro lado, están aquellas que analizan el tema más a fondo y desarrollan los ejes anteriormente planteados. Algunas de estas desarrollan los dos ejes y otras en cambio se centran en uno de ellos en especial. 
El primer grupo de fuentes da cuenta de la gravedad del problema. No solo del problema de VIF en Colombia y de los altos índices de este tipo de violencia en nuestro país, sino también del problema de la poca investigación y el bajo desarrollo literario sobre el tratamiento de salud física y mental en este tipo de violencia. Justamente estas fuentes, dentro de las cuales vale la pena mencionar la revista Forensis, recomiendan a las instituciones gubernamentales tomar medidas al respecto. Medidas que van encaminadas sobre todo a generar políticas públicas, informar a la ciudadanía sobre las herramientas que tienen para prevenir la VIF y capacitar mejor al personal de salud para atender tanto a las víctimas como a los victimarios.

De acuerdo con el estudio Beatriz María Molina Vélez: su legado a la terapia familiar en Colombia: in memoriam (Molina, 2009 ); las revistas Forensis de los años 2002, 2004 y 2007, y el documento desarrollado por el Ministerio de Protección Social de Colombia, Ilamado Modelo de atención integral en salud para víctimas de violencia sexual, el tratamiento que se le dé a este problema en los centros de salud, junto con el que se da desde el área jurídica y desde las políticas públicas, es una herramienta fundamental para acabar dos problemas: el de las víctimas inmediatas y el de los espectadores.

De hecho, algo en lo que concuerda la literatura es el grave problema del círculo vicioso que genera la VIF. Una de las características comunes de los agresores y los agredidos es que han crecido en hogares en los que hubo presencia de VIF, lo que evidencia un problema de repetición de conductas. Esto lleva a pensar que un grave problema poco abordado es el de los espectadores de VIF, pues si no reciben tratamiento podrían convertirse en futuros agresores o agredidos. Precisamente por eso es que este es uno de los actores que se trata en el segundo eje y que se estudia más adelante.

Las fuentes analizadas evidencian la poca literatura que hay sobre el tema; la razón es que es un problema que no está del todo dimensionado, ya que hay una muy baja denuncia de los casos (Instituto Nacional de Medicina Legal, 2007) de VIF, al cual se le deberían dedicar mayores recursos (Instituto Nacional de Medicina Legal, 2002) y sobre los cuales debe haber una mayor educación tanto de la ciudadanía como del personal que los va a tratar.

Así que de estas primeras recomendaciones generales, en el sentido de que no plantean modelos específicos o políticas públicas concretas sino lineamientos generales, se puede concluir que es un problema que ha sido subdimensionado por los bajos índices de denuncia. Es por esto que se necesita un mayor esfuerzo interdisciplinario para lograr una mayor capacitación del personal, tanto de salud como de policía, y así tratar y remitir correctamente a las víctimas de este tipo de violencia. De manera general, las recomendaciones que se hacen son "promocionar centros de atención especializados sobre el maltrato y el tipo de tratamiento o rehabilitación que toda víctima requiera en un momento determinado" (Instituto Nacional de Medicina Legal, 2004), y una mayor destinación de recursos para tratar de manera correcta el tema. 
Luego de exponer las recomendaciones generales, procedemos a analizar el desarrollo de la literatura en cada uno los ejes mencionados: el primero se ocupa temporalmente de las diferentes etapas que se pueden analizar en estos casos de violencia y, el segundo, de los actores que intervienen en cada una de estas etapas.

En primer lugar se abordará la prevención, a continuación la etapa del tratamiento posterior al hecho violento y en cada una se analizará los diferentes actores que intervienen.

\section{ETAPAS}

\section{A. Primera etapa: La prevención}

Como ya se había mencionado uno de los problemas más graves que envuelve la VIF es el círculo vicioso que se genera a raíz de esta. Uno de los factores de riesgo existentes en las familias es que los padres hayan crecido en familias en donde existió violencia. Esto los hace más vulnerables para que en un futuro reproduzcan el patrón y se conviertan en agresores o agredidos. Por esto mismo es que cada vez se le da más importancia a la prevención y se presta más atención al cuidado y desarrollo de esta etapa. Fuentes como la revista Forensis (2007 y 2008), Barragán (2002) y González (2009), intentan tomar este y todos los elementos de riesgo y tratarlos de manera temprana para que se logre reducir el número de casos de VIF.

La literatura sobre esta etapa trata por lo tanto programas institucionales y políticas públicas de educación, ya que considera que estos son los medios idóneos para prevenir el problema de la VIF. La educación se da en dos ámbitos; por un lado, la educación de las víctimas o posibles víctimas de este tipo de violencia para que conozcan cómo actuar frente a un hecho violento dentro de la familia y cómo detenerlo o pedir ayuda para hacerlo. Por otro lado, educación del personal de salud y otro tipo de personal que puede estar involucrado con víctimas de VIF, que deben aprender a tratarlas y saber a dónde remitirlas para que reciban la ayuda necesaria. De este segundo grupo hacen parte el personal de las comisarías de familia y la policía nacional, entre otros.

La revista Forensis de 2010 propone, además de la educación, otras tres herramientas para mitigar la violencia en la familia. Estas son redes, comités, programas de protección y apoyo y legislación. Algunos de los elementos importantes que se deben tener en cuenta al implementar cada una de las acciones anteriormente mencionadas son las características y contextos específicos de cada territorio en el que se pretendan implementar, ya que la idea es que se apliquen en espacios como las escuelas, las familias y la comunidad en general (Ministerio de Protección Social, 2011).

Es plausible concluir, a partir de las fuentes analizadas, que en lo que respecta a la prevención, la atención se ha dirigido a la producción de políticas públicas o esfuerzos interdisciplinarios, más que a la elaboración de textos de literatura que aborden el tema. Se trata de "agrupar entidades gubernamentales y no gubernamentales, 
así como grupos comunitarios, en función de potenciar esfuerzos hacia un objetivo común" (Barragán, 2002). Esto puede deberse a que la naturaleza del problema impulsa a que se busquen herramientas más de tipo práctico que teórico.

Una de las políticas públicas citada en varias de las fuentes analizadas, que vale la pena mencionar, es "Haz paz", una política pública del Instituto Colombiano de Bienestar Familiar (ICBF), la Consejería Presidencial de Programas Especiales, la Consejería Presidencial para la Equidad de la Mujer, la Defensoría del Pueblo, el Departamento Administrativo de Bienestar Social del Distrito y el Departamento Nacional de Planeación (DNP). Esta es una política nacional de construcción de paz y convivencia familiar que busca prevenir la VIF desde tres perspectivas: desde un cambio cultural; desde el fortalecimiento de los factores protectores de la familia, la pareja y el individuo; y desde el fortalecimiento de los factores protectores comunitarios. Estos tres niveles se ven de manera similar en otras fuentes (Gónzalez, 2009, p. 251) donde por ejemplo se establece un nivel comunitario, un nivel familiar y un nivel personal. Se busca por lo tanto un apoyo integral y para ello se requiere de esfuerzos mancomunados de muchas instituciones y partes de la comunidad.

A nivel comunitario se busca informar y capacitar en "la identificación de situaciones de maltrato y orientación hacia las instituciones que brindan protección a la familia" (Gónzalez, 2009, p. 251), informar a la familia sobre cuáles son las consecuencias de la existencia de vio- lencia dentro de la familia, educar en las formas de construir convivencia comunitaria, buscar espacios de participación social y de diálogo. A nivel familiar se busca fortalecer la integración familiar, la confianza, el ejercicio diario de derechos, deberes y responsabilidades; la comunicación abierta entre todos los integrantes de la familia. A nivel personal se busca el autocontrol y la tolerancia. Y a nivel cultural un cambio de la sociedad y de las instituciones.

Para llevar a cabo todo lo anterior, Barragán (2002) propone una serie de estrategias intersectoriales que son la estrategia política, la educativa, la de comunicación y una de promoción del buen trato. En la estrategia política "se busca el posicionamiento del problema en la agenda política y presupuestal" (2002) y se busca darle mayor importancia institucional y así conseguir mayores recursos para un mejor desarrollo de todos los esfuerzos que buscan atacar el problema. En la estrategia educativa se busca capacitar a "maestros, padres de familia, estudiantes y personal de salud como multiplicadores del buen trato" (2002). En la estrategia de comunicación se persigue la creación de canales internos y externos para que las redes funcionen adecuadamente. Por último, la estrategia de promoción del buen trato incluye acciones como núcleos de reflexión, talleres de capacitación y jornadas de movilización social.

De lo anterior podemos concluir que en la etapa de prevención son relevantes dos cosas: primero, la capacitación a todos los agentes que actúan en esta etapa del tratamiento, tanto a los agredidos como a la comunidad en general, al 
personal de salud y al personal de atención; y, segundo, que para que se logre una efectiva prevención es necesaria la acción interdisciplinaria.

\section{B. Segunda etapa: El tratamiento posterior al hecho violento}

En esta segunda etapa, libros como Beatriz María Molina Vélez: su legado a la terapia familiar en Colombia: in memoriam (Molina, 2009), Encuentros regionales de redes de prevención y atención a la violencia intrafamiliar/M. Antioquia (Dirección Seccional de Salud de Medellín, 1997), y El cuidado de enfermería en la salud mental del ciclo evolutivo humano (González, 2009), se han ocupado de cuáles son las características que debe tener el personal de salud para atender a las víctimas y victimarios de la VIF, así como los procedimientos que se deben llevar a cabo en estos casos. Primero, hay que tener en cuenta que esta etapa a su vez tiene varias subetapas o niveles que son: el prediagnóstico e intervención inicial, el terapéutico y el especializado (Romero, 2009). El objetivo común que buscan todas estas medidas es una mayor eficacia del sistema de salud. Con una mayor capacitación del personal y unos procedimientos más claros es posible agilizarlos y a la vez lograr un efectivo mecanismo de seguimiento de los casos. A continuación se explicará de forma más detallada cada uno de los niveles.

\section{Prediagnóstico e intervención inicial}

Esta primera etapa consiste en el primer acercamiento que hace una víctima de VIF o un agresor a las instituciones de salud. Lo que señala la literatura existente sobre el tema es establecer cuál es el procedimiento que deben seguir los proveedores de salud y cuáles son los elementos a los que debe prestarse suma atención para que la atención brindada sea de mejor calidad.

Hay un punto relevante que se debe tener en cuenta y es la capacitación del personal de los centros de atención. El personal debe ser capacitado incluyendo todos los niveles de atención mencionados. Las personas deben ser recibidas por personal apto que pueda ayudarlas, para detectar posibles agresiones domésticas, aun cuando las víctimas no lo confiesen, y que puedan remitir de forma adecuada a las víctimas o victimarios que ya han aceptado su condición. Es sumamente importante que el personal que esté a cargo de las historias médicas esté preparado para diligenciarlas, ya que esta información es muy valiosa y servirá no solo para hacer un diagnóstico adecuado sino también para poder detectar otros casos que se encuentren dentro de la misma familia (Restrepo, 2006, p. 12)

\subsection{El personal de los centros de atención frente a los agresores}

El personal de salud que se encarga de atender este tipo de casos debe, como primera medida, establecer si está tratando con el agresor o con la víctima para poder determinar cuál será el tipo de atención que se brindará y el proceso que se debe seguir. Cuando se está frente a los agresores es importante tener en cuenta medidas adicionales. La primera medida que debe tomar el personal del centro de salud es analizar los posibles riesgos de que el paciente reaccione de manera violenta y para ello debe 
realizar una evaluación a distancia (Escobar et al., 2011).

Luego debe hacerse una entrevista inicial en donde se recomienda que el tratante se identifique, empiece la entrevista con temas neutros, hable en forma clara, calmada y repetitiva; siempre deje claro que tiene deseos de ayudar; busque la cooperación del paciente; debe dejarlo hablar y ponerle atención a todo lo que diga. Además es recomendable que tenga presente la importancia de intervenciones no verbales que también debe adoptar, como no dar la espalda, no tocar al paciente, mantener las manos a la vista, tener una distancia prudente, no realizar movimientos bruscos y usar un tono de voz calmado (Escobar et al., 2011).

Después, dependiendo del caso, se pasa a una intervención verbal, es decir, a "un diálogo enfocado a generar tranquilidad y autocontrol en el paciente, realizado por personal capacitado en el área" (Escobar et al., 2011); un diagnóstico probable; la inmovilización mecánica o la sedación farmacológica (Escobar et al., 2011).

Lo anterior está relacionado con pacientes que llegan en estado de alteración a los centros de atención, que es como usualmente se llega a conocer y a poder tratar a los agresores. Lo que nos lleva a otro problema existente en Colombia evidenciado por las fuentes estudiadas y es el problema de la poca existencia de infraestructura adecuada para atender este tipo de pacientes (Escobar et al., 2011).

El tratamiento a los agresores es de suma importancia porque de esta forma ellos pueden asumir la responsabilidad frente a su comportamiento y a las consecuencias del mismo dentro de la familia. El evaluador debe indagar acerca de la: "expectativa del agresor y de la relación con la víctima; tipos de comportamiento y tácticas abusivas o maltratantes; riesgos para niños y niñas; $y$ factores de riesgo que pueden incrementar el nivel de riesgo o peligrosidad". (Restrepo, 2006)

\subsection{El personal de los centros de atención frente a las víctimas}

El primer punto que se tiene que mencionar en este apartado es la respuesta a la pregunta: ¿quiénes son los agredidos o las víctimas? Según las fuentes analizadas se ha considerado que son tres los grupos de la población que son vulnerables a sufrir ataques de violencia dentro de la familia. Estos son las mujeres, los niños y los adultos mayores (Restrepo, 2006, p. 12).

Sin embargo, el artículo "Imaginarios sobre violencia conyugal de hombres víctimas, que han solicitado orientación a los líderes afectivos del proyecto Medellín convive en familia" (Correa, 2008), buscó reevaluar esta tesis al establecer que debido a los cambios sociales y culturales de los últimos años, la mujer ha adquirido un nuevo rol dentro y fuera de la familia y esto ha ido de la mano con un aumento en los casos de VIF con víctimas hombres (Correa, 2008). Lo cierto es que en el imaginario colectivo los hombres no son uno de los grupos vulnerables y por eso muchas veces es aun más difícil obtener denuncias de este grupo poblacional. Por eso dentro de las fuentes encontradas ninguna hace referencia directa al tratamiento que 
debe llevarse a cabo cuando las víctimas son hombres.

En todo caso, se ha establecido que uno de los problemas más graves que envuelve este fenómeno es la falta de denuncia, es por eso que el personal de salud se encuentra frente a una difícil situación a la hora de acercarse a los pacientes. Para empezar el tratante debe decidir cómo enfrentar a los pacientes que Ilegan. Hay quienes optan por hacer una evaluación inicial de manera rutinaria sin importar si son casos sospechosos o no, otros por el contrario prefieren hacer las preguntas de manera directa solo en los casos que consideran que están en riesgo de sufrir agresiones. Las referencias bibliográficas revisadas, como Correa (2008) y Restrepo (2006), presentan los dos mecanismos como opciones, no existe una respuesta correcta y una errada, todo depende del caso y de la actitud que el tratante considere más efectiva. Esto debe estar acompañado de capacitación para analizar cuáles son los casos considerados sospechosos y prestar mayor atención a ellos.

Por lo anterior, la entrevista y evaluación inicial debe ser hecha por un proveedor de salud que se haya formado en teorías y dinámicas sobre VIF, seguridad, derechos y autonomía de víctimas. Tiene que haberse entrenado para indagar sobre el tema y para brindar información acerca de los recursos, las competencias y las redes de atención. Debe tener autorización para llenar documentos oficiales y técnicos sobre la historia clínica y por último debe haber establecido lazos de confianza con el paciente, para esto se recomienda que nunca se le culpe o se le obligue a demostrar su condición de víctima y no mostrar ningún tipo de sesgo o prejuicio (Correa, 2008). Por último es importante que el proveedor de los servicios de salud nunca le insista al paciente que debe alejarse del agresor ya que debe respetarse totalmente la autonomía del paciente.

\subsection{El personal de los centros de atención frente a los espectadores}

En el caso de los espectadores hay grandes dilemas que las fuentes revisadas analizan. El primero de ellos es el de establecer en qué momento observar agresiones de los miembros de la familia se vuelve en sí mismo un caso de VIF. El segundo es si una vez que se sabe que existe un caso de abuso infantil, se debe alejar al niño de la familia y enviarlo a un hogar de paso o no. Esto es un problema porque no siempre todos los miembros de la familia representan un peligro para el menor y no siempre es la mejor solución para su situación. La respuesta que dan las fuentes analizadas (Restrepo, 2006, p. 28) es que este debe ser el último recurso que debe tomarse, ya que esto también genera consecuencias negativas para el menor. El tercero es en qué forma debe hacerse la evaluación inicial; es recomendado que el proveedor de salud la haga a solas con el paciente pero muchas veces esto no es posible con los niños, porque los adultos que están a cargo de ellos pocas veces acceden a esto y nunca pasará con los menores de tres años. Por lo anterior, el personal de salud debe ser perspicaz para identificar los casos de violencia y no pasar por alto ningún indicio. Debe tener especial cuidado con todos los signos y síntomas de maltrato o abandono. 


\section{Nivel terapéutico}

Una vez se ha analizado el nivel de prediagnóstico se estudiará el segundo nivel que es el terapéutico, la función de la terapia familiar es "la atención a las relaciones intra y extrafamiliares con las personas directamente implicadas en la crisis (dinámica familiar, estructura, límites, roles, funciones, comunicación, autoridad y autonomía). La intervención es en el grupo familiar". (Dirección Seccional de Salud de Medellín, 2002).

En Colombia la terapia familiar ha tenido un desarrollo de varias etapas (Molina, 2009). En sus orígenes se usaba el modelo de la víctima y el verdugo (Molina, 2009), que normalmente era el hombre que se encontraba en un lugar superior de dominio y poder. En esta etapa el tratamiento era objetivo, es decir, se determinaban las razones del pasado que llevaban a la aceptación pasiva de la situación. El hombre era excusado por la estructura social y no entraba en el proceso de ayuda terapéutica. En una segunda etapa, se incorporaron nuevas teorías que invitaban a ver el todo y no solo las partes. En esta etapa se entendía la VIF como una "red de relaciones con varios participantes, cuestionando el modelo víctima-verdugo y mirando la situación dentro de un paradigma circular" (Molina, 2009).

En la tercera etapa, la terapia empieza a incluir fenómenos externos como la raza y la orientación social y se empieza a abordar desde dos polos: las condiciones sociales y la conducta individual con énfasis en el desarrollo del ape- go y la intimidad. El tratamiento debe hacerse en varios niveles. Por un lado, un tratamiento individual para cada uno de los miembros de la familia en violencia no severa; cuando la violencia sea más severa los hombres perpetradores deben asistir a un tratamiento grupal; por último, una ayuda especializada a los niños (Molina, 2009).

Para llevar a cabo la terapia familiar se debe tener en cuenta primero que el riesgo de VIF está presente en todas las familias y por eso se debe preguntar de manera directa, una vez confirmada la existencia de VIF, y lo que se busca es solucionar el problema directamente. Para la evaluación se debe tener un conocimiento de la naturaleza de este tipo de violencia, el impacto en la vida de los actores y el contexto en el que opera. Se recomienda que se dé espacio para la participación voluntaria de las personas, tener respeto por lo que cada persona diga que será confidencial hasta que decidan hablarlo en conjunto, se sugiere que se adopte un modelo de coterapia, con un equipo o supervisión que disminuyan el miedo y la rabia (Molina, 2009).

Los temas que deben tratarse son: los detalles de la conducta violenta, la responsabilidad de cada uno y lo inapropiado de cada conducta y se deben crear límites y un plan realizado en conjunto para evitar la violencia durante el tratamiento. Para que se lleve a cabo un tratamiento en conjunto debe haber acuerdo entre las dos partes; debe tratarse de casos de violencia menor o medianos, en los que haya ausencia de riesgos mortales, de lo contrario debe hacerse terapia a cada una de las partes por separado. 


\section{Nivel especializado}

Acerca de este último nivel no hay mucho desarrollo. Lo que se busca es que la capacitación del personal de salud que recibe los casos de VIF, y que les da la asistencia primaria necesaria, pueda remitir a las víctimas y agresores adecuadamente y que les pueda brindar la información necesaria para que ellos reciban el tratamiento interdisciplinario del que se habló anteriormente.

Una muestra de lo anterior son las redes de apoyo social, que buscan modificar el comportamiento de las personas frente a un problema determinado. Este mecanismo en conjunto con los líderes de apoyo logran que "una vez la familia o la persona afectada logra hacer un reconocimiento de su problema, la red de apoyo social y en particular el líder se movilizan para brindarle ayuda" (Dirección Seccional de Salud de Medellín, 1997). Luego de escuchar y brindar apoyo, la red brinda información y orienta a la familia o a la persona para que busque ayuda profesional o legal. Si es necesario, la red o el líder acompaña a la familia o persona a la institución competente. Por último, la red cumple una etapa de seguimiento de la situación (Dirección Seccional de Salud de Medellín, 1997).

\subsection{El caso particular de la ruta de atención en violencia sexual}

Dentro de este nivel hay un documento que merece un especial análisis, se trata de la Ruta de atención intersectorial de las violencias sexuales en Medellín, realizado por la Secretaría de
Bienestar Social de la Alcaldía de Medellín, en el 2007. Si bien no trata específicamente de la VIF, sí alude a esta en varias ocasiones y además es un modelo muy completo que puede implementarse en los casos de VIF con las adecuaciones pertinentes. Según este documento, la violencia sexual requiere, por un lado, de una intervención intersectorial para la construcción de propuestas integrales de atención y reglamentación de los delitos sexuales; dicha atención debe realizarse tanto para víctimas como para agresores. Y, por el otro, de una difusión de la educación desde una perspectiva de restablecimiento de derechos que implica una promoción y una prevención.

Hay cuatro sectores gubernamentales que deben participar en la ruta y que tienen la responsabilidad de posicionar el tema como un tema de salud pública en sus intervenciones. Estos son salud, justicia, protección y educación. Desde cualquiera de estos sectores donde se reciba el caso, comienza la ruta de atención. De ahí que la importancia sea saber reconocer en cada caso específico a dónde debe direccionarse. Así que es sumamente importante que todos los sectores "se apoyen y conversen entre sí" (Alcaldía de Medellín, 2007, p. 2) para buscar la adecuada articulación.

Dentro de las dificultades que se encontraron vale la pena resaltar la poca capacitación sobre la normatividad, así como la falta de unidad de criterios sobre cómo aplicarla, y los problemas para unificar la clasificación y el reporte de los casos para la vigilancia y el control. En el caso concreto de la VIF se identificó que la eficacia 
de la atención depende de la sensibilización y del compromiso de quienes atienden, por eso es necesaria la unificación de los criterios de categorización, clasificación y lineamientos de acción en la atención de las víctimas de VIF (Alcaldía de Medellín, 2007, p. 4).

Otro elemento de este documento que vale la pena resaltar es que no se limita a poner en evidencia la falta de denuncia sino que además afirma que es el Estado el encargado de suplir la demanda de servicio y tener las ofertas adecuadas para poder resolver los casos denunciados. Lo anterior por la importancia que tiene en estos procesos la denuncia, ya que es la única forma de evitar que los hechos se perpetúen y que se busque una solución. Por eso la ruta parte precisamente de la denuncia, de donde se lleva a la asesoría, la consultoría y la atención.

La ruta de atención que plantea el documento tiene cinco pasos que son: “(0) Detección: en las comunidades y sensibilizar para la denuncia social, civil; (1) Recepción y direccionamiento (inicio de proceso institucional); (2) Diagnóstico (verificación de pruebas a nivel sectorial); (3) Tratamiento (reparación, manejo intersectorial); (4) Evaluación y seguimiento" (Alcaldía de Medellín, 2007, p. 6).

De lo anterior se puede concluir que la importancia de este documento desarrollado por la Alcaldía de Medellín, tiene como elemento fundamental la intención de realizar un esfuerzo interdisciplinario para la atención a los casos de violencia sexual. Esto resulta relevante porque busca que los diferentes entes que inter- vienen se articulen unificando criterios y procedimientos para brindar una mejor atención a las víctimas.

\section{CONCLUSIONES}

A partir del análisis anterior se pueden sacar tres grandes conclusiones. La primera es el poco desarrollo que existe en Colombia acerca del tratamiento de VIF en el campo de la salud física y mental. A pesar de los altos índices que existen en nuestro país de este tipo de violencia, el tema del tratamiento ha sido relegado. Esto genera muchos vacíos ya que de nada sirve un gran desarrollo legal e institucional si desde el sector de la salud física y mental no se está generando desarrollo y control para mejorar la problemática. Además, muchas de las iniciativas se crean de manera independiente y desestructurada lo que genera la existencia de dobles esfuerzos en el mismo sentido. Es decir, por la falta de apoyo y de trabajo interdisciplinario los pocos esfuerzos que se están haciendo no están siendo efectivos.

La segunda es que dentro del tema del tratamiento, otra cuestión muy importante es el trabajo que lleva a cabo el personal de los centros de atención. Este está envuelto en grandes problemas éticos por lo delicado del tema que trata. Por eso es importante hacer énfasis en que debe ser un personal calificado. Se debe tener especial cuidado con no tener prejuicios, no presionar a la víctima, no culparla, respetarla y respetar sus decisiones y, sobre todo, ser discreto con la información confidencial que maneja. De esta 
manera se crea un ambiente de mayor confianza con la víctima y la ayuda será más efectiva.

Asimismo, el personal debe estar en capacidad de llenar las historias clínicas y otros documentos importantes que se necesiten. Dentro de este punto se debe mencionar el manejo de evidencias. Los proveedores de salud deben saber documentar y cuidar debidamente todos aqueIlos elementos que puedan servirle a la víctima como evidencia del ataque. De esta manera, la víctima podrá hacer uso de las herramientas legales con mayor facilidad y así nuevamente será más efectiva la ayuda interdisciplinar que se le puede brindar a la víctima.

La tercera es que todavía hay muchos vacíos para lograr que los servicios prestados sean integrales y sean de cobertura nacional. Para tener servicios de este tipo que sean más efectivos y exitosos estas son algunas de las recomendaciones: deben existir resguardos y centros de protección, líneas telefónicas para intervención de crisis, intervención y terapia de crisis, grupos de apoyo, servicios clínicos y mentales de atención inmediata y remisión para intervención psicológica, asesoría y acompañamiento en el proceso legal, centros de atención como consejería y terapia vocacional, servicio de reubicación de domicilios, transporte a las víctimas para que puedan acceder a los servicios, planeación de acciones para la protección y seguridad integral conjuntamente con el sector de protección y justicia, servicios de apoyo a los espectadores de VIF (Restrepo, 2006).
La VIF es una problemática que tiene muchas caras y dimensiones, así que su solución no puede estar solamente en el área de la salud como tampoco en el área del derecho, sino que debe darse de manera conjunta, así se reduce la posibilidad de error, se tiene mayor certeza sobre el diagnóstico y se da una atención integral. Además, tampoco se puede solucionar el problema atendiendo solo uno de los actores, es decir, no solamente atendiendo a las víctimas ni solamente a los victimarios. La interdisciplinariedad es una salida plausible para atender a todos los actores de una manera más completa para romper la repetición de patrones que se crea en el tipo de familias que sufren este tipo de violencia, en donde la mayoría de espectadores de estos episodios tienden a repetir estos patrones al crecer.

\section{Bibliografía}

Alcaldía de Medellín, Secretaría de Bienestar Social. Comité Coordinador para la Política Pública en Prevención y Atención de las Víctimas de las VSx en Medellín. (2007). Ruta de atención intersectorial de las violencias sexuales-VSx en Medellín.

Asociación Colombiana de Hospitales y Clínicas (ACHC). (2007). Violencia sexual en Colombia. Parte de hospitalaria (Bogotá). Asociación Colombiana de Hospitales y Clínicas (ACHC), 9 (55).

Barragán, F. (2002). Experiencia intersectorial, interinstitucional e interdisciplinaria para abordar el maltrato infantil y la violencia do- 
méstica. Congreso Internacional de Derecho de Familia. Conflictos familiares, su prevención y tratamiento. Bogotá: Universidad Externado de Colombia.

Correa, D. M., López, CH. A. y Álvarez, M. P. (2008). Imaginarios sobre violencia conyugal de hombres victimas, que han solicitado orientación a los líderes afectivos del proyecto "Medellín convive en familia" del municipio de Medellín 2005 -2007. Medellín.

Dirección Seccional de Salud, Medellín. Dirección Seccional de Salud de Antioquia. (1997). Encuentros regionales de redes de prevención y atención a la violencia intrafamiliar/ M. Antioquia.

Dirección Seccional de Salud. Medellín. Dirección Seccional de Salud Antioquia. (2002). Modelo de intervención en crisis para casos de violencia intrafamiliar y violencia sexual en: Herramientas para adquirir una buena salud mental. Antioquia.

Dirección Seccional de Salud. Medellín. Dirección Seccional de Salud de Antioquia. (2002). Herramientas para adquirir una buena salud mental. Antioquia.

Dirección Seccional de Salud de Antioquia. Dirección de Salud Pública. (2006). Evaluación del sistema de vigilancia en salud pública de la violencia intrafamiliar. Antioquia, 2002-octubre de 2006. Parte de la información para la Acción (pp. 1-7). Recuperado de http:// www.dssa.gov.co/index.php/documen- tos-de-interes/vigilancia-epidemiologica/ bias/2006/757-bia-noviembre-2006/file

Escobar, F., Suárez, M. F. (2011). Abordaje clínico del paciente violento en atención primaria. Universitas Médica Bogotá, 52 (4), 421-430.

Eslava, C. (1994). Aspectos generales de la terapia de familia y terapia de familia en maltrato. Bogotá: Asociación Colombiana para la Defensa del Menor Maltratado.

González, T. C. (2009). El cuidado de enfermería en la salud mental del ciclo evolutivo humano. Santa Marta: Universidad del Magdalena.

Instituto Colombiano de Bienestar Familiar (ICBF). (Acciones en torno a promoción, prevención, detección, atención integral y vigilancia de la violencia intrafamiliar desde $y$ para servicios del sector salud: documentos conceptual. Recuperado en http://es.scribd. com/doc/37182348/Documento-Marco1-Acciones-Sector-Salud\#download

Instituto Forense de Medicina Legal y Ciencias Forenses. Centro de Referencia Nacional sobre Violencia. (2002). Datos para la vida: herramienta para la interpretación, intervención y prevención del hecho violento en Colombia/ MR.Forensis. 2003. Bogotá.

Instituto Forense de Medicina Legal y Ciencias Forenses. Centro de Referencia Nacional sobre Violencia. (2004). Datos para la vida: herramienta para la interpretación, intervención y prevención del hecho violento en Colombia/ MR. Forensis. 2004. Bogotá. 
Instituto Forense de Medicina Legal y Ciencias Forenses. Centro de Referencia Nacional sobre Violencia. (2007). Datos para la vida: herramienta para la interpretación, intervención y prevención del hecho violento en CoIombia/ MR. Forensis. 2007. Bogotá.

Jaramillo, D. E. (2001). Rol del personal de salud en la atención a las mujeres maltratadas. Parte de investigación y educación en enfermería: Facultad de Enfermería Universidad de Antioquia (Medellín), 19 (1), 38-45.

Londoño, A. (2001). Derecho a los derechos: atención integral a sobrevivientes de delitos sexuales. Bogotá: Fondo de Población de las Naciones Unidas (FNUAP).

Ministerio de Protección Social. (2011). Modelo de atención integral en salud para víctimas de violencia sexual. Bogotá.

Molina, B. M. (2009). Beatriz María Molina Vélez: su legado a la terapia familiar en Colombia: in memoriam. Medellín: Todo GráficasFundación Bienestar Humano.

Restrepo, O. L. (2006). Modelos de atención para la prevención, detección y tratamiento de la violencia doméstica. Documento marco para orientar: "Acciones en torno a promoción, prevención, detección, atención integral y vigilancia de la violencia intrafamiliar desde y para servicios e instituciones del sector salud". Bogotá: Instituto Colombiano de Bienestar Familiar (ICBF). Recuperado en http:// es.scribd.com/doc/37182348/DocumentoMarco-1-Acciones-Sector-Salud

Restrepo, O. L. (2006). Guías para detección, evaluación, atención y seguimiento a personas y familias en situación de víctimas o espectadores de violencia intrafamiliar desde instituciones prestadoras de servicios de salud. Bogotá: Instituto Colombiano de Bienestar Familiar (ICBF).

Romero, V. M. y Amar, J. (2009). Modelo de atención integral a mujeres, niñas y niños víctimas de violencia intrafamiliar llevado a cabo en centros de atención de la ciudad de Barranquilla (Colombia). Parte de Investigación y desarrollo, 17 (1).

Saldarriaga, L. M. (2007). Datos y voces del buen vivir: evaluación de impacto cualitativa: proyecto buen vivir: atención psicoterapéutica. Medellín: Alcaldía de Medellín, Secretaría de Bienestar Social. 